\title{
Study for Rapid Determination of Tetrodotoxin Based on Lateral Flow Immunoassay
}

\author{
Jun $\mathrm{CHEN}^{1}$, Jing $\mathrm{ZHAO}^{3}$, Ning-Ping $\mathrm{TAO}^{3}$ and Jian $\mathrm{CONG}^{*}, 2$ \\ ${ }^{1}$ Shanghai No.4 Reagent \& H.V.Chenmical Co., LtD. Shanghai, 200940, China \\ ${ }^{2}$ College of Urban Public Security Administration, Shanghai Open University, Shanghai, 200433, \\ China \\ ${ }^{3}$ College of Food Science and Technology, Shanghai Ocean University, Shanghai, 201306, China \\ *Corresponding author, email:Jcong@sou.edu.cn
}

Keywords: Tetrodotoxin; Lateral flow immunoassay, Superparamagnetic nanoparticle probe, Rapid detection.

\begin{abstract}
Trichloroacetic acid (TCA) was used as a protein precipitation in the pretreatment process. The content of TCA in the sample was optimized. Lateral flow immunoassay (LFIA) based on superparamagnetic nanoparticle (SPMNP) probe for TTX detection was employed as a detection method. This article is intended to establish a fast, easy, effective TTX detection method and a matching pretreatment method that could be applied to on-site test. It is found that the obtained results were identical with the reference analytical values after the sample treated by $12 \%$ TCA and with 5:1:1 ratio of $10 \%$ Tween-20 in BS to $2 \mathrm{~g} / \mathrm{mL}$ BSA in BS to $0.35 \mathrm{~g} / \mathrm{mL}$ sucrose in BS combined. The detection limit of TTX-spiked samples was found to be $6 \mathrm{ng} / \mathrm{mL}$. The whole assay time of the qualitative test for TTX detection was less than 1.5h.
\end{abstract}

\section{Introduction}

Tetrodotoxin (TTX), $\mathrm{C}_{11} \mathrm{H}_{17} \mathrm{~N}_{3} \mathrm{O}_{8}$, named by Tahara in 1909, is a kind of non-protein neurotoxins with high toxic, low molecular weights, also as a kind of typical sodium ion channel inhibitor. TTX can be soluble in weak acid, slightly soluble in water and strong acid, insoluble in organic reagents.

Puffer fish meat, liver and testis are welcomed food in China and Japan. But it was reported that general cooking methods could hardly destroy its toxicity with stable chemical property [1]. Lots of people lost their lives from TTX poisoning and lack of relief measures every year. Nowadays, food safe becoming more and more popular allows quick and efficient TTX detection method developing.

Recent decades have seen the rapid development and application of immunoassays in the field of modern detection area. Lateral flow immunoassay (LFIA) is extensive application based on Enzyme-linked immuno sorbent assay (ELISA) with high sensitivity and accuracy, simplicity, inexpensive and operating convenience. The main study on LFIA focuses on using colloidal gold as tracer in recent years [2].

In order to develop a rapid, simple and highly specific detection method for TTX detection, a competitive assay based on the principle of immunoassay using nano magnetic polymer materials instead of traditional materials as tracer was studied. In this study, specific monoclonal antibodies against TTX were prepared and a magnetic LFIA technology was innovatively used to realize rapid qualitative and quantitative detection of the analyses.

\section{Materials and Methods}

Reagents and apparatus TTX standard (95\% purity) was purchased by Hebei Aquatics Research Institute (Hebei, China). Acetic acid, trichloroacetic acid (TCA), sodium hydrate, disodium hydrogen phosphate, sodium dihydrogen phosphate, sodium tetraborate, boric acid (analytical pure) were purchased from Sinopharm Chemica Reagent Co., Ltd. (Shanghai, China). Sodium azide, 
sucrose, Tween-20, 1-ethyl-3-[3-dimethylaminopropyl] carbodiimide hydrochloride (EDC), N-Hydroxysuccinimide (NHS), 2-[N-morpholino] ethane sulfonic acid (MES), bovine serum albumin (BSA, Albumin Fraction V), Tris base and BCA (bicinchoninic acid) protein quantitation kits were purchased from Sangon Biotech Co., Ltd. (Shanghai, China). All the solutions were prepared with distilled water.

A MAR Assay Development System was purchased from Magna Bioscience (LLC, USA) for magnetic signal reading in this paper. SPMNPs (carboxyl group modified, 200nm) were purchased from Quantum Design Co., Ltd. (Beijing, China). Materials for strips construction, including CN 140 (Sartorius stedim Biotech, Germany) used as nitrocellulose membrane, absorbent pad, conjugate pad, support sheet, top cover film and test cassettes were purchased from Shanghai Jiening Biological Technology Co., Ltd. (Shanghai, China). Goat anti-mouse secondary antibody (GAM) was purchased from Longene Biotech Co., Ltd. (Hangzhou, China).

ELISA kit was purchased from Beijing Zhongwei Food Hygienic Technology Co., Ltd (Beijing, China). Deposable PD-10 desalting columns and protein G affinity chromatography column (GE Healthcare, USA) were purchased from Beijing Zhongtian Jingwei technology Co., Ltd. (Beijing, China).

Puffer fish (Takifugu obscurus and Takifugu flavidus) including muscle, liver, skin, kidney, intestine, gonad were purchased from Zhongyang group Co., Ltd (Jiangsu, China).

TTX extraction In this study, TCA was chosen as deproteinization reagent to eliminate the protein in the sample solution. 100\% TCA solution was added to supernatant slowly until the final TCA concentration reached. The sample pretreatment process is shown in Fig.1.

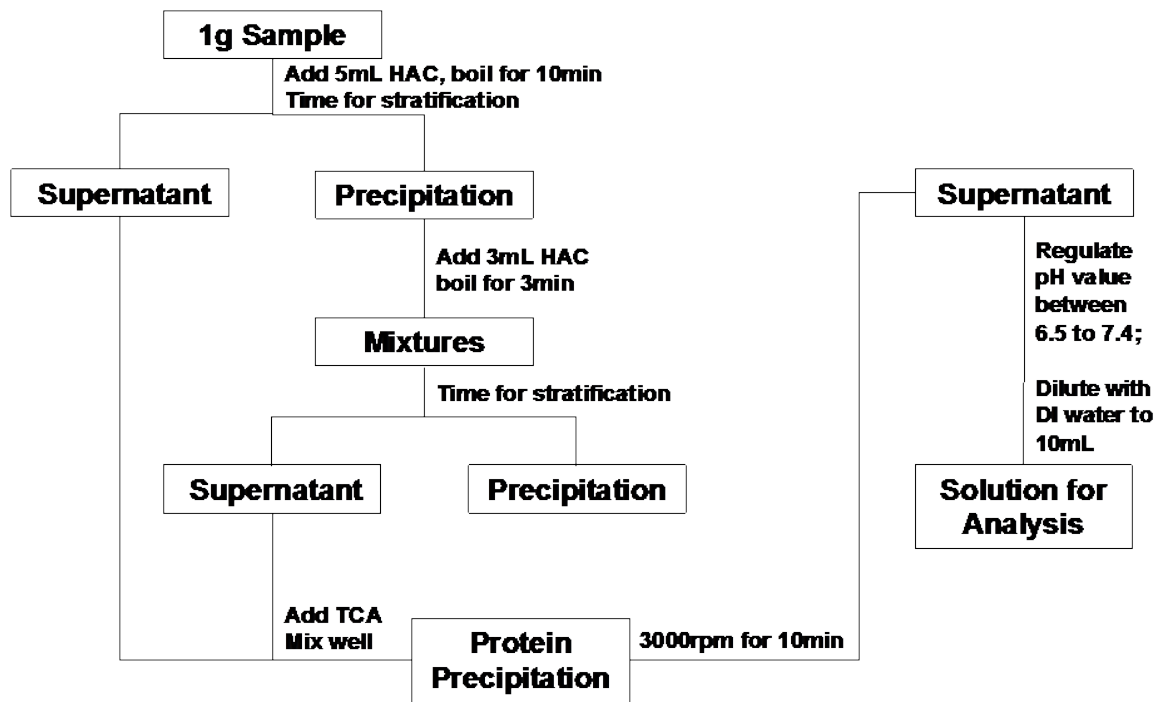

Fig.1 Flowchart of pretreatment of protein precipitation by TCA

The tissue was placed into the test tube after taking $1 \mathrm{~g}$ tissue from sample fish. Secondly, $5 \mathrm{~mL}$ HAC was added into the tube before placing the tube in the water bath and boiled for 10min. Thirdly, the supernatant was taken by pipettor after several minutes for stratification. Then, 3mL HAC was added into the remaining and boiled for another 3min. The steps for taking supernatant above should be repeated 2 times. After that, the supernatant and add 100\% TCA solution were collected into the test tube. The test tube was put into the centrifugal machine, centrifuged at $3000 \mathrm{rpm}$ for $10 \mathrm{~min}$. The $\mathrm{pH}$ value of supernatant solution was adjusted between 6.5 7.4 by $\mathrm{NaOH}$ after centrifugation and diluted with DI water to $10 \mathrm{~mL}$ at last.

Different levels of TCA (2\%, 5\%, 8\%, 10\%, 12\% and 15\%) in the solution were adopted.

In order to evaluate the results of the pretreatment process, BCA protein quantitation kits were used to detect the concentration of protein residues after the processing.

Antigen and antibodies Tetrodotoxin immunogen (TTX-BSA) employed in this study was prepared by a modified formaldehyde method. Firstly, mix up $600 \mu \mathrm{L}$ of TTX $(0.6 \mathrm{mg}$ in $0.1 \%$ HAC) 
and $150 \mu \mathrm{L}$ of BSA $(3.0 \mathrm{mg}$ in $10 \mathrm{mM}$ PBS), $1: 5$ mass ratio of TTX to BSA. Then, $37 \%$ formaldehyde was added dropwise till the final concentration was $4.4 \%$. The mixture was vertically mixing for $72 \mathrm{~h}$ at room temperature. Finally, unreacted reagent, residual free TTX in the mixture was removed by the desalting column (PD-10 GE) against $10 \mathrm{mM}$ PBS (pH 7.4) at $4{ }^{\circ} \mathrm{C}[3$, 4].

5H10 Monoclonal Antibodies (McAbs) against TTX was prepared and characterized in our previous work. $5 \mathrm{H} 10$ hybridomas were intraperitoneally injected to BALB/c mice to prepare ascites. The ascites were purified by following steps: concentrating twice by ammonium sulfate, desalting by a deposable desalting column, affinity purification by a protein $G$ affinity chromatography column, and at last, desalting again. Purified 5H10 McAbs were freeze-dried, re-dissolved in 10 mM PBS (pH 7.4) [5].

GAM secondary antibody was diluted with deionized water to $1 \mathrm{mg} / \mathrm{mL}$

\section{Super Paramagnetic Nanoparticle-Based LFIA for TTX Detection}

Preparation of TTX specific SPMNP probe: Firstly, $66.7 \mu \mathrm{L}$ carboxyl-modified SPMNPs (Original concentration was $2.8 \%$, about $2 \mathrm{mg}$ ) was placed in the test tube. Mix up after $500 \mu \mathrm{L}$ MEST buffer $(0.01 \mathrm{~mol} / \mathrm{L}$, pH5.0, MES buffer containing $0.05 \%(\mathrm{v} / \mathrm{v})$ Tween-20) was added. Place the test tube in the magnetic separation bed to remove the supernatant. Repeat above three steps three times. Then, $2 \mu \mathrm{L}$ EDC $(0.5 \mathrm{~g} / \mathrm{ml})$ and $4 \mu \mathrm{L}$ NHS $(0.25 \mathrm{~g} / \mathrm{ml})$ were mixed with the SPMNPs suspension (diluted to $500 \mu \mathrm{L}$ ), and reacted with SPMNPs for 30min. After the SPMNPs was washed with BST buffer $(0.005 \mathrm{~mol} / \mathrm{L}$, pH9.0, containing $0.05 \%(\mathrm{v} / \mathrm{v})$ Tween-20) for three times, $120 \mu \mathrm{g} 5 \mathrm{H} 10 \mathrm{McAbs}$ were added into SPMNPs suspension and reacted for 3h at room temperature. Next, 1\% BSA was added into the SPMNPs suspension to block the active sites of the nanoparticles through physical adsorption. After reacting for 30min, the obtained specific SPMNPs probes were finally dispersed in the conservation solution ( $\mathrm{pH} 9.0,0.005 \mathrm{~mol} / \mathrm{L} \mathrm{BST}, 0.05 \%$ Tween-20, $1 \% \mathrm{NaN} 3,0.1 \% \mathrm{BSA}$ ), and stored at $4^{\circ} \mathrm{C}$.

Structure of LFIA test strip: The test strip employed in this study was composed by support sheet, sample pad, conjugate pad, absorbing pad and nitrocellulose (NC) membrane (CN 140), as shown in Fig.2. Striped NC membrane $(300 \mathrm{~mm} \times 25 \mathrm{~mm})$, conjugate pad $(300 \mathrm{~mm} \times 10 \mathrm{~mm})$, sample pad $(300 \mathrm{~mm} \times 20 \mathrm{~mm})$, and absorbing pad $(300 \mathrm{~mm} \times 25 \mathrm{~mm})$ were pasted onto the support sheet $(300 \mathrm{~mm} \times 100 \mathrm{~mm})$ with a transparent top cover pasted on the membrane as a protection sheet. TTX-BSA $(0.05 \mathrm{mg} / \mathrm{mL})$ and GAM $(1 \mathrm{mg} / \mathrm{mL})$ were sprayed onto NC membrane (CN 140) at the rate of $0.8 \mu \mathrm{L} / \mathrm{cm}$, respectively as Line $\mathrm{T}$ and Line $\mathrm{C}$. At last, the assembled sheet was cut into $5 \mathrm{~mm}$-wide strips for detection after the NC membrane dried [6].

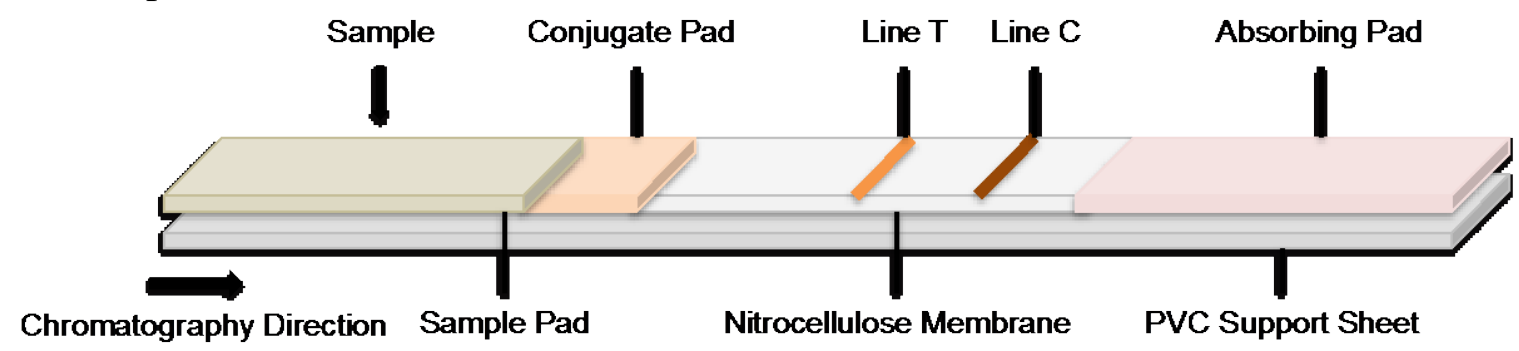

Fig.2 Composition of Lateral flow immunoassay test strip

Lateral flow test procedure: At first, $4 \mu \mathrm{L}$ SPMNPs probe suspension was applied on the conjugate pad. After that, mix up the $105 \mu \mathrm{L}$ pretreated sample and $15 \mu \mathrm{L}$ proposed additive consisted of various ingredients. Then, $120 \mu \mathrm{L}$ prepared sample was added at a slowly constant speed on the surface of the sample pad for TTX detection. Magnetic signals values at Line T and Line $C$ could be detected by a MAR system after immunochromatography reaction for about 30min at room temperature.

Optimization of LFIA system: Different concentrations of BSA, sucrose and Tween-20 
combined with various ratios were discussed.

The pretreatment process in this study involved using TCA for protein precipitation. And the concentration of TCA solution would be closely related to the magnetic signal strength. Six concentrations of TCA $(2 \%, 5 \%, 8 \%, 10 \%, 12 \%$ and $15 \%)$ were respectively tested according to the procedure mentioned above.

Determination: The test strip was fed into the specialized cassette after the immunochromatography finishing. Then, the cassettes were sent into the instrument for MAR signal analysis.

Establishing calibration curve: In order to determine the TTX concentration in the sample precisely, TTX standard was added into the negative sample. Standard curve was established by using the magnetic signal value ratio of TTX standard to negative as ordinate, and the corresponding logarithm values of TTX concentration $(\mathrm{ng} / \mathrm{mL})$ as abscissa.

Quantitative calculation of puffer fish samples: Abscissa values (logarithm values of TTX concentration) can be got in the calibration curve according to the magnetic signal value ratio of sample to negative. TTX concentration $(\mathrm{ng} / \mathrm{mL})$ can be obtained by calculating antilogarithmic values. TTX content is set by the formula (Eq.1):

$$
\text { TTX content }(\mu \mathrm{g} / \mathrm{g})=\mathrm{C} \times \mathrm{K} / 1000 \mathrm{~m}
$$

Where C donates the concentration of TTX (ng/mL) in the diluted extracts, $\mathrm{K}$ the dilution multiple, $\mathrm{m}$ the weight (g) of the sample.

Mouse unit (MU) is a common standard used to judge the toxicity [7]. 1MU equals to the concentration of $0.22 \mu \mathrm{g} / \mathrm{g}$. The values of mouse unit are classified into three levels: "Negative": <10MU. "Weakly positive": >10MU and <100MU."Positive": >100MU and <1000MU. "Highly toxic": >1000MU.

\section{Performance Index of the LFIA System}

Recoveries of method with varied amounts of TCA adopted: TTX standard was added into the sample tissue at $100 \mathrm{ng} / \mathrm{mL}$ in this part. To determine the optimal proportion of TCA in the sample supernatant, TCA was added at different levels of 2, 5, 8, 10, 12 and 15\%. The recovery of standard addition was calculated.

Recoveries of method with varied addition of TTX standard: In this part, TTX standard was added into the sample tissue at three different levels of 10, 100 and 200ng/mL. The proportion of TCA in the sample was fixed. Recoveries were calculated.

Stability for LFIA analysis: The test of repeatability was conducted by repeating the experiment for five times to get the relative standard deviation (RSD). TTX standard was put into the sample tissue at three different levels of 10,100 and $200 \mathrm{ng} / \mathrm{mL}$ with the proportion of TCA in the sample supernatant fixed.

LFIA sensitivity: The calculating method of LFIA sensitivity made reference to the formula admitted by IUPAC (International Union of Pure and Applied Chemistry) and ACS (American Chemical Society).

$$
\mathrm{C}_{\mathrm{L}}=\left(\mathrm{K} \times \mathrm{S}_{\mathrm{B}}\right) / \mathrm{m}
$$

Where CL donates the concentration of the limit of detection, $\mathrm{K}$ the constant, SB the standard deviation of blank value, $\mathrm{m}$ the sensitivity shown in slope of linear regression equation. 


\section{Comparison and Evaluation Study on LFIA and ELISA}

The LFIA results were evaluated in using of commercially available ELISA kits. The samples treated by TCA were applied to the ELISA kit. Process and methods of ELISA kit according to the product's operation manual were as follows.

Pretreatment method: Weigh $5 \mathrm{~g}$ fish muscle and cut to pieces. Keep in boiling water for 10min after $0.1 \%$ acetic acid $(25 \mathrm{~mL})$ was added. After being cooled to room temperature, filter paper was adopted to filter the supernatant. Then, $0.1 \%$ acetic acid $(20 \mathrm{~mL})$ was added into the precipitation, boiled for 3min. After being cooled to room temperature, the mixture and the supernatant were collected to the centrifugal tube, centrifuged at $3000 \mathrm{~g}$ for $10 \mathrm{~min}$ to remove the tissue. The same volume chloroform was added to the solution, shook for $1 \mathrm{~min}$. Gather the liquor after stratification. Repeat that steps. After removing the chloroform, the solution was adjusted to pH6.5-7.4. Dilute with PBS solution to $50 \mathrm{~mL}$ and preserved at $4^{\circ} \mathrm{C}$.

Pretreatment method: Rinse the plates with wash fluid for $3 \mathrm{~min}$, repeat twice. Add $50 \mu \mathrm{L}$ standard or sample into each wells. Then, add the TTX monoclonal antibodies into wells and incubated for $90 \mathrm{~min}$ at $37^{\circ} \mathrm{C}$. Rinse the plates with wash fluid for $3 \mathrm{~min}$, repeat three times. Add $100 \mu \mathrm{L}$ HRP conjugate into the wells and incubated for $60 \mathrm{~min}$ at $37^{\circ} \mathrm{C}$. Rinse the plates with wash fluid for $3 \mathrm{~min}$, repeat five times. Add $100 \mu \mathrm{L}$ colorimetric solution and incubated for $12 \mathrm{~min}$ at $37^{\circ} \mathrm{C}$. Add $50 \mu \mathrm{L}$ terminative reagent and detecting every pore's absorbtion in wavelength of $450 \mathrm{~nm}$.

\section{Results and Discussion}

Calibration curve for TTX detection External standard method was used to establish a calibration curve for quantitative analysis. New standard curve should be made once the conditions of the experiment changed.

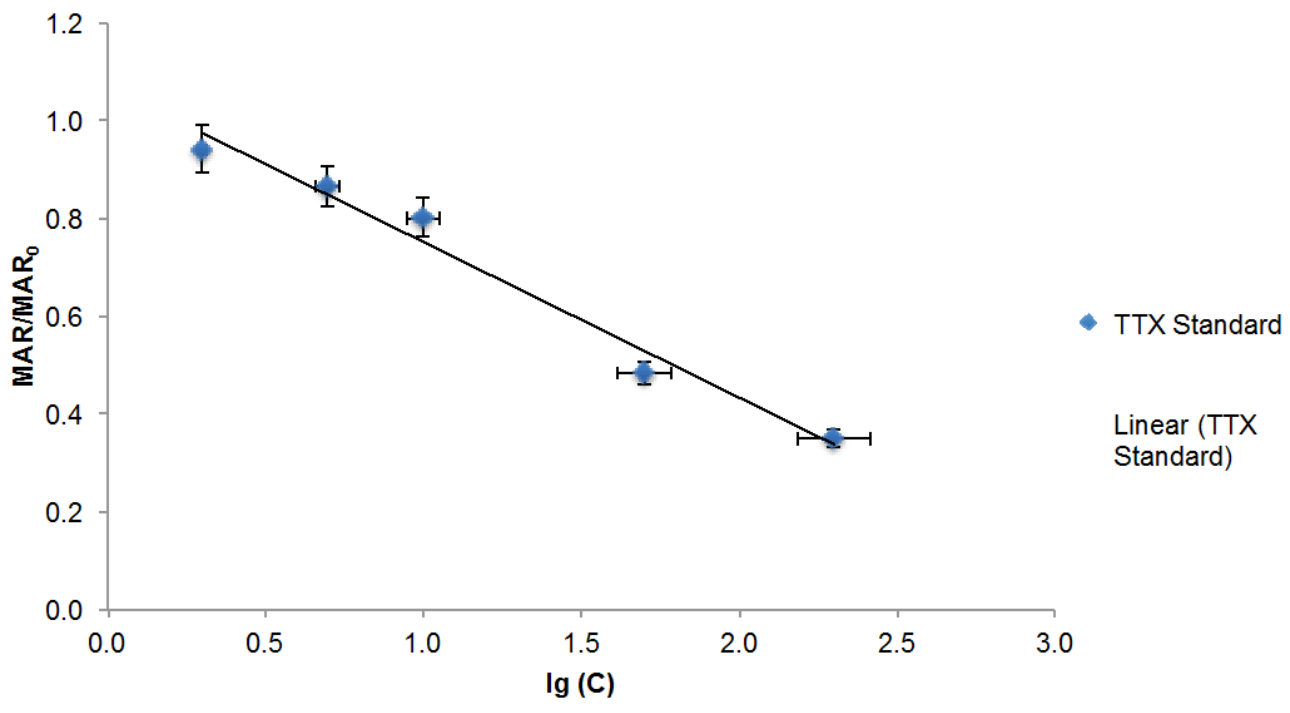

Fig.3 Calibration curve for TTX detection

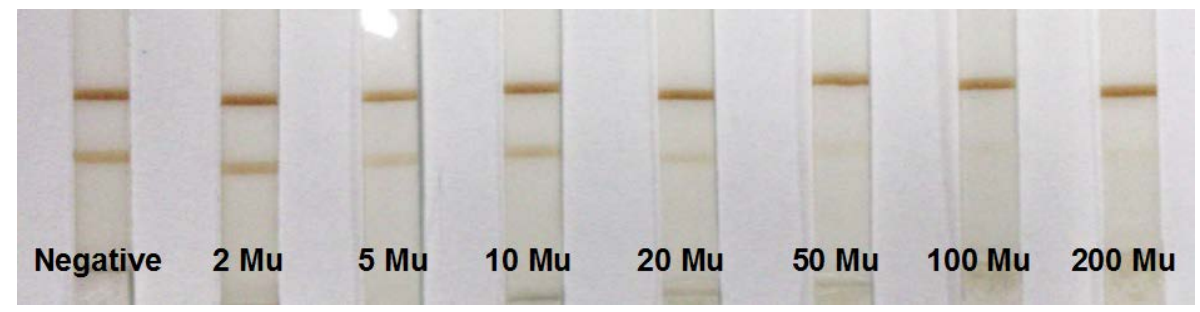

Fig.4 Illustrate visible band in test strips at various TTX concentrations

The equation between the magnetic signal value ratio of TTX standard and the corresponding 
logarithm values of TTX concentration (ng/mL) is set by the formula (Eq.3). The calibration curve liner ranges for TTX quantitative detection is $4.4 \mathrm{ng} / \mathrm{mL} \sim 4400.0 \mathrm{ng} / \mathrm{mL}$, with a linear correlation coefficient of 0.9771 .

$$
y=-0.3186 x+1.0703
$$

With the increasing of TTX mouse unit values in sample (0 200MU), the depth of color showed at Line $\mathrm{T}$ changed from clearly visible to invisible (Fig.4).

Effect of TCA on protein denaturation TCA is a kind of reliable protein precipitant. In order to find the proper concentration of TCA solution to make albumen denaturation, the BCA protein quantitative assay was performed after six concentrations of TCA (2\%, 5\%, 8\%, 10\%, 12\% and15\%) were respectively adopted in the pretreatment process according to the procedure mentioned above. And the results of the protein quantitative assay were shown in Table 1.

Table 1 BCA protein quantitation results of samples treated by various concentrations TCA

\begin{tabular}{cccc}
\hline $\begin{array}{c}\text { TCA proportion in sample } \\
(\%)\end{array}$ & $\begin{array}{c}\text { OD }_{562} \\
\text { Mean } \pm \mathrm{sd}\end{array}$ & $\begin{array}{c}\text { CV } \\
(\%)\end{array}$ & $\begin{array}{c}\text { BCA protein quantitative assay results } \\
(\mu \mathrm{g} / \mathrm{mL})\end{array}$ \\
\hline 2 & $1.205 \pm 0.079$ & 13.4 & 669.125 \\
5 & $0.993 \pm 0.043$ & 12.1 & 536.313 \\
8 & $0.787 \pm 0.004$ & 1.9 & 407.875 \\
10 & $0.746 \pm 0.001$ & 7.2 & 382.250 \\
12 & $0.695 \pm 0.022$ & 6.1 & 350.063 \\
15 & $0.619 \pm 0.008$ & 8.7 & 302.563 \\
\hline
\end{tabular}

Samples from two phases of the pretreatment process were employed to investigate the effects of TCA adopted in this pretreatment method. One was obtained after twice processed with acid and heat. The other was taken after the processing finished. The protein quantitation for the supernatant of twice processed with acid and heat reached $1421.42 \mu \mathrm{g} / \mathrm{mL}$.

As shown in the Table 1, the concentration of the protein residues in the sample supernatant showed a descending trend basically with the content of TCA in sample solution increasing. It indicated that using TCA could effectively remove the protein by comparing the BCA protein quantitative assay result of no TCA treated sample and TCA treated sample. Also, we could find that, protein quantitative assay result showed an obvious changing trend to stable with TCA proportion in the sample solution increasing.

Effect of TCA on LFIA system In this study, the experiment couldn't be carried out without the sample condition adjusted. The ingredients of the sample such as the level of ions, $\mathrm{pH}$ values, the content of heteropolymeric proteins, lipids and saccharides would have a great impact on the immunochromatography testing results [8-10]. Some agents that could promote the chromatography should be added into the sample such as the surfactant and so on [11]. The experiment suggested that $5: 1: 1$ ratio of $10 \%$ Tween-20 in BS to $2 \mathrm{~g} / \mathrm{mL}$ BSA in BS to $0.35 \mathrm{~g} / \mathrm{mL}$ sucrose in BS was suitable. The sample solution should be blended with the mixture in proportion before the sample solution added on the sample pad for detection.

The levels of magnetic signal values would be influenced by every composition in the extracts. Added standard sample tissue was treated by different amounts of TCA in order to find out the most optimized TCA content which could lead to an ideal magnetic signal level. The TTX standard was diluted to $100 \mathrm{ng} / \mathrm{mL}$ with tissue extractions. The extracts were blended with the mixture mentioned above before test. The results were shown in Table 2.

Table 2 LFIA test strip results of standards treated by various concentrations TCA

\begin{tabular}{cccc}
\hline $\begin{array}{c}\text { TCA proportion in sample } \\
(\%)\end{array}$ & $\begin{array}{c}\text { MAR signal values at Line T } \\
\text { Mean } \pm \text { sd } \\
(\mathrm{RMU})\end{array}$ & $\begin{array}{c}\text { cv } \\
(\%)\end{array}$ & $\begin{array}{c}\text { LFIA results } \\
(\mathrm{ng} / \mathrm{mL})\end{array}$ \\
\hline 2 & $804.5 \pm 52.6$ & 13.4 & 121.3 \\
\hline
\end{tabular}




\begin{tabular}{cccc}
\hline 5 & $1886.2 \pm 24.3$ & 1.9 & 2.3 \\
8 & $1486.7 \pm 40.0$ & 12.1 & 10.0 \\
10 & $1030.6 \pm 21.5$ & 7.2 & 53.1 \\
12 & $778.8 \pm 1.4$ & 6.1 & 133.2 \\
15 & $665.4 \pm 41.9$ & 8.7 & 201.5 \\
\hline
\end{tabular}

The MAR signal values are in close relationship with the performance of detection system. High and low level of the magnetic signal value would cause low sensitivity. With the increasing of TCA proportion in sample (2 15\%), the magnetic signal values were listed in Table 2, where the signal from strips with TCA proportion in sample at $12 \%$ showed the satisfactory value. The relative standard deviation (coefficient of variation) for determination of samples was all below 15.0\%.

The results in the Table 1 and 2 both presented that the TCA content in sample solution of 12\% seemed more appropriate for the sample pretreatment. At this level of TCA adopted, the protein residual amount was acceptable. Also, at this level, the detection system for TTX could be possible to obtain higher sensitivity since the magnetic signal was at an ideal value. MAR signal values at Line $\mathrm{T}$ of $100 \mathrm{ng} / \mathrm{mL}$ added standard treated by $15 \%$ proportion of TCA was much lower which caused low sensitivity, although the sample treated by TCA of $15 \%$ could remove even more protein residues. Last but not least, the TTX test result was close to the TTX standard added amount. Taken together, $12 \%$ TCA was considered the optimal approach to conduct the further studies under this condition.

Besides, TCA was believed to have roles in slower immunochromatography speed, aggregating the SPMNP at Line T, and enhancing the MAR signal, however, it also might cause the false positive.

Method validation TTX standards were added into the muscles of the puffer fish by pipettor at designed concentrations. The TTX in the fish sample was determined by LFIA after being pretreated by $12 \%$ TCA. Under optimized conditions, the results were shown in Table 3 with an excellent reproducibility of method. The RSD values were all less than $15 \%$. The recovery of the standard addition at levels of 10 and $100 \mathrm{ng} / \mathrm{mL}$ was relatively high. It could be due to the effect of TCA on the protein. Meanwhile, with the increase of the concentration of the TTX standards added, the interference of TCA became smaller.

Table 3 Detection of TTX added sample by LFIA

\begin{tabular}{ccccccccccc}
\hline $\begin{array}{c}\text { Concentration } \\
\text { of TTX } \\
(\mathrm{ng} / \mathrm{mL})\end{array}$ & 1 & 2 & 3 & 4 & 5 & $\begin{array}{c}\text { MAR signal } \\
\text { values at } \\
\text { Line T } \\
\text { Mean } \pm \text { sd } \\
\text { (RMUs) }\end{array}$ & $\begin{array}{c}\text { LFIA } \\
\text { results } \\
\text { Mean } \pm \\
\text { sd }\end{array}$ & $\begin{array}{c}\text { Recovery } \\
(\%)\end{array}$ & $\begin{array}{c}\text { RSD } \\
(\%)\end{array}$ \\
10 & 1367.8 & 1341.2 & 1347.8 & 1430.1 & 1277.2 & $1352.8 \pm 54.9$ & 16.6 & 166.4 & 4.1 \\
100 & 777.9 & 778.2 & 780.4 & 805.4 & 778.2 & $784.0 \pm 12.0$ & 130.8 & 130.8 & 1.5 \\
200 & 754.5 & 756.5 & 671.5 & 646.2 & 578.9 & $681.5 \pm 75.5$ & 195.9 & 98.0 & 11.1 \\
\hline
\end{tabular}

In this study the constant K used to determine the sensitivity of competitive immunoassays was 2 . The limit of detection was $6 \mathrm{ng} / \mathrm{mL}$.

Effect of TCA on ELISA The added standards were applied to ELISA kit after treated by TCA. Table 4 showed the results.

Table 4 Detection of TTX added sample by ELISA kit

\begin{tabular}{cccccccc}
\hline $\begin{array}{c}\text { Concentration of TTX } \\
(\mathrm{ng} / \mathrm{mL})\end{array}$ & 1 & 2 & 3 & $\begin{array}{c}\text { OD }_{450} \\
\text { Mean } \pm \mathrm{sd}\end{array}$ & $\begin{array}{c}\text { ELISA results } \\
\text { Mean } \pm \text { sd } \\
(\mathrm{ng} / \mathrm{mL})\end{array}$ & $\begin{array}{c}\text { Recovery } \\
(\%)\end{array}$ & $\begin{array}{c}\text { RSD } \\
(\%)\end{array}$ \\
\hline 10 & 0.611 & 0.497 & 0.604 & $0.571 \pm 0.064$ & 58.8 & 588.2 & 11.2 \\
100 & 0.564 & 0.565 & 0.565 & $0.565 \pm 0.001$ & 58.6 & 58.6 & 0.1 \\
200 & 0.489 & 0.477 & 0.466 & $0.477 \pm 0.012$ & 88.6 & 44.3 & 2.4 \\
\hline
\end{tabular}

Table 4 showed that TCA had strong influence on the results of ELISA, but the effects were 
relatively fixed. The testing results were in proportion to the order of magnitudes of TTX concentration. TCA might be fixed effect on the antigens coated in the ELISA plate.

\section{Comparison between ELISA and SPMNP based LFIA for TTX Detection}

The researches in immunoassay for TTX detection are currently being focused in China and Japan. ELISA method is studied the most. National standard for TTX detection by ELISA method have been set in China. In this study, we compared the SPMNP based LFIA test strips with the commercial ELISA kits in the following aspects. a. The limit of detection for ELISA is $6 \mathrm{ng} / \mathrm{mL}$ which is lower than $10 \mathrm{ng} / \mathrm{mL}$ for LFIA. b. The data obtained in this work also exhibit that it is more applicable to on-sight test. Time needed in the whole process of LFIA test strips including preparation was around half of ELISA kits took (LFIA need about $1 \sim 1.5 \mathrm{~h}$ ). In the measurement process, the operation steps were much easier, without requiring the use of any other special equipment. Results could be direct observation by eyes after sampling. c. The test strips help to preserve and easy to carry. It could be kept one year under normal temperature. Conversely, ELISA kits should be frozen in 4 degrees.

Moreover, the proteins would precipitate out during adjusting $\mathrm{pH}$ after treating by frequently used pretreatment method of ELISA[12]. It might be that happens to reach the isoelectric point when the $\mathrm{pH}$ value of the extracts was changed, an effect that made the proteins dissolved out. However, this phenomenon would never occur if TCA could be used during the course of preparation.

The lower time consuming of LFIA was partly due to the TCA adopted that could drastically reduce the test cycles. Meanwhile, TCA could meet the requirements of the pretreatment for SPMNP based LFIA. However the result showed that the sample applied to ELISA after treating by TCA might be subject to an inaccuracy consequence. Of the samples tested and compared between two methods (Table 3 and Table 4), the recoveries were affected more notable when using ELISA to detect. This could result from antigens and antibodies being destroyed by TCA residues in the sample during the incubation time. Nevertheless, TCA residues in the sample would not affect the antigens coated on the test strip because of less reaction time.

Application of TTX detection The results of LFIA test strips were evaluated by the results of ELISA kit as reference (Table 5). The pretreatment method followed the ELISA kit operation manual.

Table 5 Results of different tissues of puffer fish detected by test strips and ELISA kits

\begin{tabular}{ccccccccc}
\hline & \multicolumn{4}{c}{ ELISA kit $(\mathrm{n}=3)$} & \multicolumn{5}{c}{ LFIA test strip $(\mathrm{n}=3)$} \\
\cline { 2 - 9 } Sample & $\begin{array}{c}\text { OD }_{450} \text { value } \\
\text { Mean } \pm \text { SD }\end{array}$ & $\begin{array}{c}\text { CV } \\
(\%)\end{array}$ & $\begin{array}{c}\text { ELISA } \\
\text { results } \\
(\mathrm{ng} / \mathrm{mL})\end{array}$ & $\begin{array}{c}\text { Toxic } \\
\text { determine }\end{array}$ & $\begin{array}{c}\text { MAR value } \\
\text { Mean } \pm \text { SD } \\
(\mathrm{RMUs})\end{array}$ & $\begin{array}{c}\mathrm{CV} \\
(\%)\end{array}$ & $\begin{array}{c}\text { LFIA } \\
\text { results } \\
(\mathrm{ng} / \mathrm{mL})\end{array}$ & $\begin{array}{c}\text { Toxic } \\
\text { determine }\end{array}$ \\
\hline 1 & $1.679 \pm 0.067$ & 4.0 & 4.50 & N.D. & $2446.5 \pm 105.7$ & 4.3 & 6.51 & N.D. \\
2 & $1.720 \pm 0.069$ & 4.0 & 4.00 & N.D. & $2823.4 \pm 106.8$ & 3.8 & 2.26 & N.D. \\
3 & $1.825 \pm 0.014$ & 0.8 & 3.00 & N.D. & $2887.7 \pm 328.7$ & 11.4 & 1.89 & N.D. \\
4 & $1.398 \pm 0.037$ & 2.6 & 10.00 & N.D. & $2212.5 \pm 167.0$ & 7.5 & 12.54 & N.D. \\
5 & $1.374 \pm 0.077$ & 5.6 & 10.80 & N.D. & $1968.7 \pm 101.2$ & 5.1 & 24.85 & N.D. \\
\hline
\end{tabular}

*N.D. means none detected because of the value of mouse unit is under 10MU.

The toxic determine results from ELISA kit generally agreed with SPMNP based LFIA's, and the quantitative results were similar within normal and acceptable range.

\section{Conclusions}

Puffer fish could only be available on the basis of edible safe guaranteed. Developing a reliable rapid detection method for TTX without destroying the edible parts by scientific means was a challenge need to face.

In this paper, pretreatment methods and competitive LFIA based on SPMNP probes for TTX were discussed. An ideal rapid detection method should contain an efficient pretreatment method 
that could meet the requirements of the system. As a protein denaturant, TCA is commonly used to change the protein conformations by exposing the hydrophobic groups [13]. The approach we took was using $100 \%$ TCA diluted by the crude extracts to $12 \%$ to denature proteins presented in the food sample and cause their aggregation and precipitation. TCA is really rare applied in the pretreatment for immunoassay detection, but always employed in the preparation for electrophoresis. The amount of TCA adopted in the pretreatment was studied in this work to establish a easier, less reagent applied, fewer time consumed, smaller sample size and much more efficient way. Meanwhile, it satisfies the requirements of LFIA, the MAR signal value could be pretty ideal. On the other hand, the qualitative and quantitative results of the LFIA could be obtained within 30min taking the advantages of simple operation. Also, it was applicable for the on-site and real-time rapid examination with a strong point of efficiency, speediness and being identified with naked eye. Furthermore, this method was applicable to different matrices and allows for the TTX detection of different food materials. Our research indicates that the pretreatment with TCA adopted and the LFIA method has good potential applications in areas such as large-scale screening or point-of-care detections for TTX.

\section{References}

[1] Wu SJ, Cui JZ, Gong QL. The microbial origin of Tetrodotoxin. Marine Sciences. 10: 83-87. (2005)

[2] Tang D, Sauceda JC, Lin Z, et al. Magnetic nanogold microspheres-based lateral-flow immunodipstick for rapid detection of aflatoxin B-2 in food. Biosensors \& Bioelectronics. 25(2): 514-518. (2009)

[3] Velasco-Arjona A, Manclús JJ, Montoya A, et al. Robotic sample pretreatment-immunoassay determination of chlorpyrifos metabolite (TCP) in soil and fruit. Talanta. 45(2): 371-377. (1997)

[4] Lonnberg M, Carlsson J. Membrane assisted isoform immunoassay. A rapid method for the separation and determination of protein isoforms in an integrated immunoassay. Journal of Immunological Methods. 246(1-2): 25-36. (2000)

[5] Zhu J, Zou N, Mao H, et al. Evaluation of a modified lateral flow immunoassay for detection of high-sensitivity cardiac troponin I andmyoglobin. Biosensors and Bioelectronics. 42(0): 522-525. (2013)

[6] Tanita T, Miyakoshi H, Nakano Y. Performance of ELISA for specific measurement of High-Molecular-Weight (HMW) adiponectin. Journal of Immunological Methods. 333(1-2): 139-146. (2008)

[7] Yin Q. Determination of Tetrodotoxin in difference tissue of pufferfish by mouse bioassay and HPLC. MS thesis, Ocean University of China, Qingdao, Shandong province, PR China (2009)

[8] Zouali M, David Stollar B. A rapid ELISA for measurement of antibodies to nucliec acid antigens using UV-treated polystyrene microplates. Journal of Immunological Methods. 90(1): 105-110. (1986)

[9] Liu RH. Research of quantitative lateral flow strip based on immunomagnetic beads. MS thesis, Shanghai Jiaotong University, Shanghai, PR China (2008)

[10] Ling TT, Yan J, Lu Y, et al. Effects of chromatography system on magnetic test paper. Jiangsu Agricultural Sciences. 40(11): 298-302. (2012)

[11] Kolosova AY, Sibanda L, Dumoulin F, et al. Lateral-flow colloidal gold-based immunoassay for the rapid detection of deoxynivalenol with two indicator ranges. Analytica Chimica Acta. 616(2): 235-244. (2008) 
[12] Gong HZ, Ji R, Jiang T, et al. Development of ELISA-kit of quantitative analysis for Tetrodotoxin. Chinese journal of Public Health. 12: 1423-1424. (2005)

[13] Jiang L, He L, Fountoulakis M. Comparison of protein precipitation methods for sample preparation prior to proteomic analysis. Journal of Chromatography A. 1023(2): 317-320. (2004) 